\title{
Importance of complex band structure and resonant states for tunneling
}

\author{
P.H. Dederichs ${ }^{\mathrm{a}, *}$, Ph. Mavropoulos ${ }^{\mathrm{a}}$, O. Wunnicke ${ }^{\mathrm{a}}$, N. Papanikolaou ${ }^{\mathrm{a}}$, \\ V. Bellini ${ }^{\mathrm{a}}$, R. Zeller ${ }^{\mathrm{a}}$, V. Drchal ${ }^{\mathrm{b}}$, J. Kudrnovskýb $^{\mathrm{b}}$ \\ a Institut für Festköperforschung, Forschungszentrum Jülich, D-52425 Jülich, Germany \\ ${ }^{\mathrm{b}}$ Institute of Physics, Academy of Sciences of the Czech Republic, CZ-18040 Praha 8, Czech Republic
}

\begin{abstract}
The paper aims at understanding the tunneling process in epitaxial magnetic tunnel junctions. Firstly, we stress the importance of the complex band structure of the insulator for the tunneling of the metal electrons. For large insulator thicknesses the tunneling current is carried by very few states, i.e., those states in the gap of the semiconductor having the smallest imaginary component of the k-vector. In the case of GaAs, $\mathrm{ZnSe}$ and $\mathrm{MgO}$ these are $\Delta_{1}$-states at the $\bar{\Gamma}$ point. Secondly, we discuss the role of resonant interface states for tunneling. Based on simple model calculations and ab initio results we demonstrate that for symmetrical barriers the minority conductance can be dominated in an intermediate thickness range by few 'hot spots' in the surface Brillouin zone, arising from resonant interface states. In these hot spots full transmission can still be obtained, when all other states are already strongly attenuated, so that the usual exponential decay can be considerably delayed. (C) 2002 Elsevier Science B.V. All rights reserved.
\end{abstract}

Keywords: Magnetoresistance; Tunneling; Band structure; Interface effects

The tunneling magnetoresistance (TMR) of magnetic tunnel junctions consisting of Ferromagnet|Insulator|Ferromagnet layers has attracted strong scientific interest, partly due to potential application as magnetic random access memories. Miyazaki and Tezuka [1] and Moodera et al. [2] were able to obtain TMR ratios up to $20 \%$ in room temperature experiments and recently room temperature values of $40 \%$ are reported by various groups. The understanding of the TMR and of the electronic structure has not progressed equally fast. Only recently ab initio calculations of the electronic structure and the spin-dependent transport have been reported [3-6].

This paper aims at gaining an insight in the tunneling through epitaxial Ferromagnet|Insulator|Ferromagnet junctions. In a recent letter [7], we demonstrated that the tunneling process through insulators can be under-

*Corresponding author. Tel.: + 49-2461/61-4351; fax: +492461/61-2620.

E-mail address: p.h.dederichs@fz-juelich.de (P.H. Dederichs). stood in terms of the complex band structure of the bulk insulator, in particular, the metal-induced gap states (MIGS) in the energy region of the gap. These results will be discussed in the first part of this paper. The second part deals with the interesting effects of resonant interface states on the tunneling, which lead to 'hot spots' in the $\mathbf{k}_{\|}$-resolved conductance and which can dominate the conductance for smaller distances.

Complex Band Structure: For out discussion we use $\mathrm{Fe} / \mathrm{I} / \mathrm{Fe}$ sandwhiches, where I refers to the semiconductors $\mathrm{Si}, \mathrm{Ge}, \mathrm{GaAs}$ and $\mathrm{ZnSe}$. In the bulk of a crystal, the periodicity demands that the Bloch $\mathbf{k}$-vectors are purely real. But near a crystal surface or interface one can match a bulk wavefunction of complex $\mathbf{k}$ with the solution outside the half-crystal $[8,9]$. For the interface between a metal and an insulator the most important of such states are MIGS, being itinerant in the metal but exponentially decaying in the insulator (for energies in the gap). Such solutions for complex $\mathbf{k}$-vectors form the complex band structure of the insulator. Although they only occur at surfaces or interfaces, they are solutions of the bulk Schrödinger equation, since the 
interface-induced changes of the potential are confined in their greatest part to the first one or two monolayers.

For the considered transport problem, under an infinitesimal applied voltage, metallic electrons with energies at the Fermi level $E_{\mathrm{F}}$ impinge on the insulator interface. Due to the assumed perfect epitaxy the inplane component $\mathbf{k}_{\|}$of the incident wave vector $\mathbf{k}=$ $\left(\mathbf{k}_{\|}, k_{\mathrm{z}}\right)$ is conserved. Therefore in the insulator only evanescent states with a given (real) energy $E_{\mathrm{F}}$ and a real $\mathbf{k}_{\|}$-component are allowed, so that only the perpendicular component $k_{z}=q+\mathrm{i} \kappa$ can be complex, with the 'decay parameter' $\kappa$ describing the exponential decrease of the wavefunction in the insulator.

The central problem for tunneling is therefore the determination of these decay parameters $\kappa$, in particular the search for the smallest possible $\kappa$-value $\kappa_{\min }$ for a given Fermi energy in the gap. In fact in the limit of large barrier thickness only the state with the smallest decay parameter, $\kappa_{\min }$, survives and contributes to the tunneling current. The determination of $\kappa_{\min }$ can proceed in two steps. Firstly, given values of $\mathbf{k}_{\|}$and $E_{\mathrm{F}}$, one has to determine, out of the infinitely many solutions with different decay parameters, the one with the smallest $\kappa>0$ value. In the second step one can then vary $\mathbf{k}_{\|}$, and search for the absolute minimum $\kappa_{\min }$ compatible with $E_{\mathrm{F}}$. Of course, states with $\kappa$ close enough to $\kappa_{\min }$ will also be important.

The method used in this work for calculation of the complex band structure is the simplest possible: we apply the local pseudopotential plane-wave technique with the empirical form factors. Since these form factors have been fitted to spectroscopic data, this method gives a reasonably good description of the band structure in the gap region, this being the most important for the tunneling. Clearly, the local density functional calculations give a much better overall description of the band structure, but they have the well-known deficiency of underestimating the band gap by about $50 \%$. Thus the above method is well-suited for our purpose.

In the following we can only briefly sketch some of our results. Fig. 1 shows the band structure of $\mathrm{ZnSe}$ for perpendicular incidence $\left(\mathbf{k}_{\|}=0\right)$ on a $\mathrm{ZnSe}\left(\begin{array}{lll}0 & 0 & 1\end{array}\right)$ interface, the real bands with $k_{z}$ real in the middle panel and the complex parts for $q=0$ in the left and for $q=$ $2 \pi / a$ in the right panel. Here directly the dispersion of the decay parameter $\kappa$ is directly plotted as a function of energy. For a given energy in the band gap the state with the smallest $\kappa$ is that of symmetry $\Delta_{1}$ (the identity representation) connecting the top of the $\Delta_{1}$ valence band to the bottom of the $\Delta_{1}$ conduction band. We expect this to be true quite generally when we are dealing with a direct gap characterized by a $\Delta_{1}$ valence band maximum and a $\Delta_{1}$ conduction band minimum. Then the loop connecting these particular extrema will be smaller than any other one, and so will be the corresponding value of the decay parameter. The next

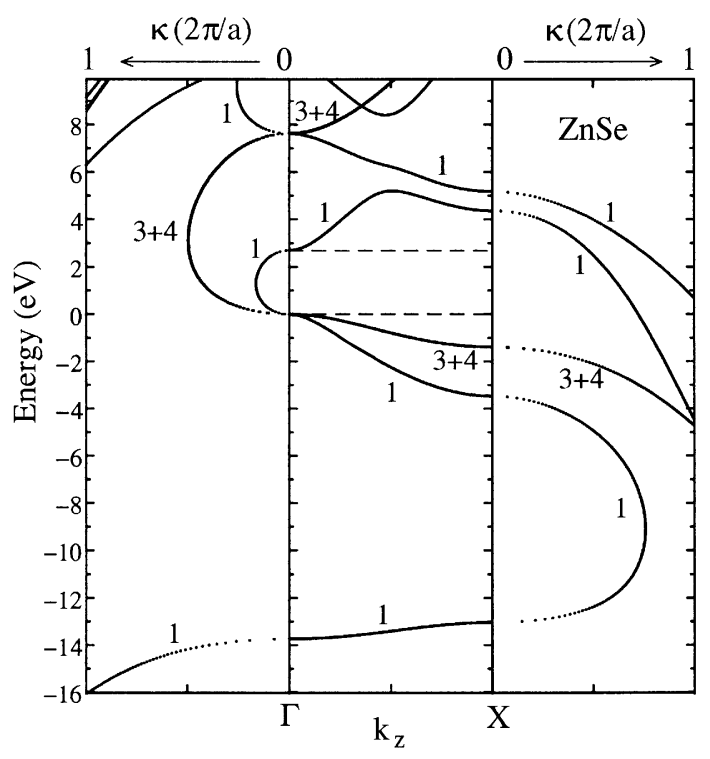

Fig. 1. The complex band structure of $\mathrm{ZnSe}$, for $\mathbf{k}_{\|}=0$, at $q=0$ (left panel) and $q=2 \pi / a$ (right panel), calculated within a local empircal pseudopotential method. The real lines are forming loops and free-electron-like parabolas.

larger $\kappa$-values arise from the degenerate $\Delta_{3}$ and $\Delta_{4}$ bands, and, additionally, infinitely many other inverseparabolic states exist starting from higher bands, having much larger $\kappa$-values.

Another illustrative example is shown in Fig. 2, the complex band structure in the $\Gamma-X$ direction for the insulator $\mathrm{MgO}$, which is known to grow epitaxially on

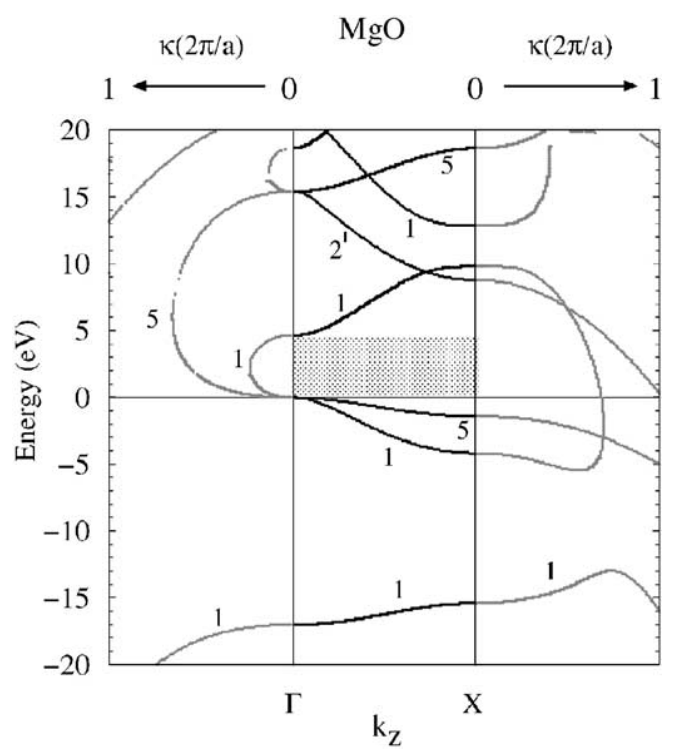

Fig. 2. The complex band structure of $\mathrm{MgO}$, for $\mathbf{k}_{\|}=0$, at $q=0$ (left panel) and $\mathrm{q}=2 \pi / a$ (right panel), calculated within the LDA by the KKR Green's function method. 
Fe. These bands have been calculated within the local density functional theory by the Korringa-KohnRostocker (KKR) Green's function method; therefore, they show the well-known error that the band gap is too small (4.6 instead of $7.8 \mathrm{eV})$. Surprisingly, the topology of the complex bands is almost the same as obtained for $\mathrm{ZnSe}$, despite the fact that the underlying crystal structures, zincblende versus rocksalt structure, are different. In particular the smallest decay constant $\kappa_{\text {min }}$ again occurs at the $\Delta_{1}$-loop at the $\bar{\Gamma}$-point.

Departing from the $\bar{\Gamma}$-point, we know that the valence states are lowered energetically, the conduction states are raised and the gap increases. For this reason it is expected that the loops at $q=0$ become larger for nonzero $\mathbf{k}_{\|}$values, and the corresponding values of $\kappa$ increase. Thus, by proceeding with the search for $\kappa_{\min }$, one can scan the whole two-dimensional surface Brillouin zone at a given energy. One would thus form a constant-energy surface in the $\left(\mathbf{k}_{\|}, \kappa\right)$-space. This consists of many branches, corresponding to the (infinitely) many different complex bands for any given $\mathbf{k}_{\|}$, but our attention should be focused on the lowestlying one. This is shown in Fig. 3 for four materials, namely $\mathrm{Si}, \mathrm{Ge}, \mathrm{GaAs}$, and $\mathrm{ZnSe}$, for a typical value of $E_{\mathrm{F}}$ in the middle of the gap. We see that in all four cases the decay parameter is minimized at the $\bar{\Gamma}$-point. We also see that the difference of $\kappa$ at the flatter part of the branch and at $\mathbf{k}_{\|}=0\left(\kappa_{\min }\right)$ increases monotonically with the band gap, this also following the order $\mathrm{Si}<\mathrm{Ge}<\cap$ $\mathrm{GaAs}<\mathrm{ZnSe}$. However other cases can also occur. For instance, for $\mathrm{Si}$ the $\kappa_{\min }$-state is determined by the complex band structure close to the X-point, if the
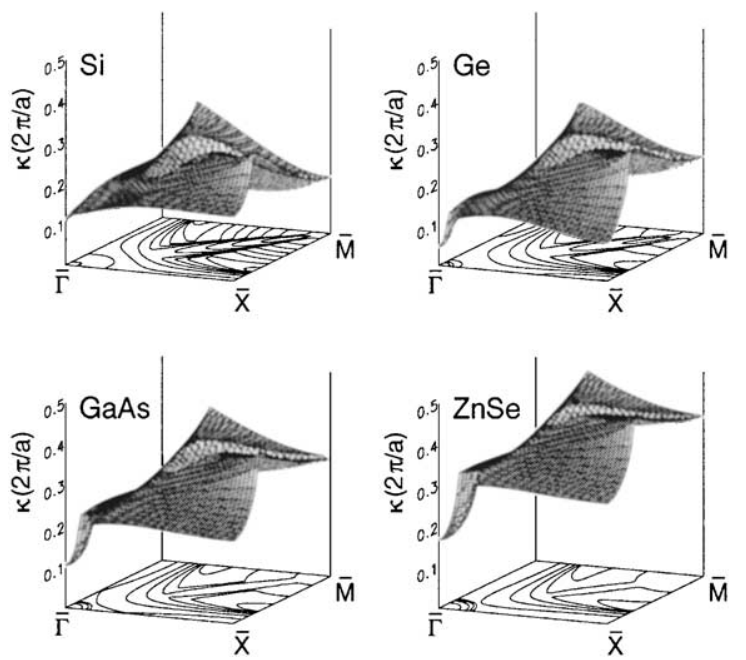

Fig. 3. The constant-energy surface $\kappa=\kappa\left(\mathbf{k}_{\|}\right)$, drawn in onefourth of the surface Brillouin zone for Si, Ge, GaAs, and ZnSe. Only the lowest positive branches of the decay parameter $\kappa$ are drawn, for an energy in the middle of the band gap. The decay parameter has its minimum at the $\bar{\Gamma}$-point in all four cases.
Fermi energy approaches the conduction band minimum, located at that point.

The above results have important consequences for tunneling. In the metal, only states of $\Delta_{1}$-symmetry (in the nomenclature of the $\mathrm{ZnSe} \mathrm{T}_{\mathrm{d}}$-symmetry group) can couple to the $\Delta_{1}$-states in the semiconductor and thus for large insulator thicknesses only metallic states at the $\bar{\Gamma}$ point and with $\Delta_{1}$-symmetry determine the tunneling currents. The different availability of these $\Delta_{1}$-states in the majority and minority bands leads to magnetoresistance. Our analysis is fully consistent with the ab initio results of MacLaren et al. [5].

In short we have shown that conclusions about the tunneling in FM/I/FM epitaxial systems can be drawn by a simple inspection of the complex band structure of the insulator in the gap region. For the above cases, this has been obtained using simple pseudopotential techniques, and as a matter of fact it can be understood, in a first approximation, just by looking at the real band structure and by noticing the symmetry of the real bands and their curvature at the minima and maxima. Thus it constitutes a very useful tool for the understanding and prediction of tunneling phenomena.

Resonant Interface States: Recent ab initio calculations of the $\mathbf{k}_{\|}$-resolved conductance of epitaxial tunnel junction show a very interesting phenomenon: for certain discrete $\mathbf{k}_{\|}$-values 'hot spots' or 'spikes' appear in the transmitted intensity, showing that electrons with such $\mathbf{k}_{\|}$-values can apparently tunnel through the junction with no or very little attenuation while all other states are very strongly damped [10-15]. This effect occurs only in the minority band of the ferromagnet and, if present, can dominate the tunnel characteristics for intermediate thicknesses. For large thicknesses, in the asymptotic limit, the behavior is determined by the complex band structure of the insulator as is discussed in this paper. An example for such hot spots is given in Fig. 4, showing the results of ab initio KKR calculations for a junction consisting of two FCC Co( 0001$)$ halfcrystals separated by 4 monolayers of vacuum. The $\mathbf{k}_{\|}$-resolved conductance is plotted in the two-dimensional (001) Brillouin zone for Co minority electrons at the Fermi level. The conductance is dominated by four double peaks in the $\bar{\Gamma}-\bar{X}$ directions, compared to which the contributions from other peaks seem to be negligible. The structure of these double peaks will be discussed later. While it is tempting to attribute the hot spots to numerical problems in the complicated evaluation of the conductance, several groups have demonstrated recently that these spots are connected with the occurrence of interface states in the minority band [10-15]. Yet details of the mechanism, in particular, why these hot spots are practically not damped, are still not properly understood, as we believe.

In this section we present a detailed investigation of the properties and the occurrence of these hot spots. We will discuss the effect by a simple analytical model and 


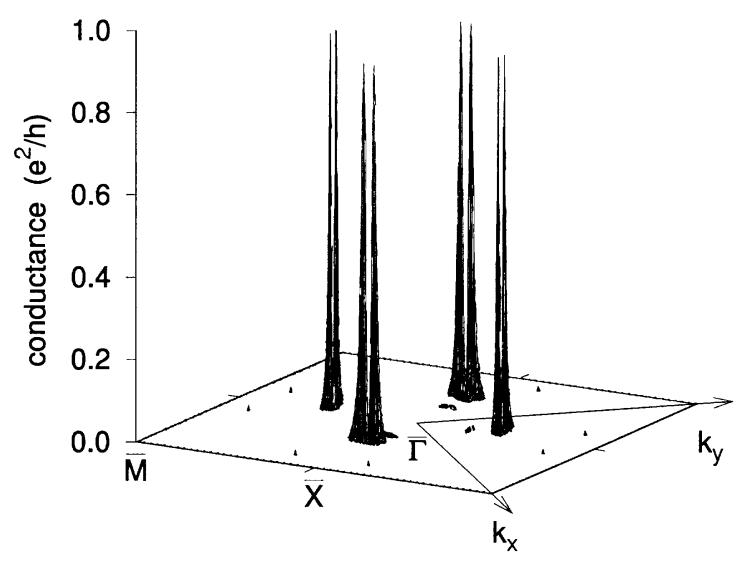

Fig. 4. $\mathbf{k}_{||-}$-resolved conductance plot in the SBZ for the minority band for a $\mathrm{Co}|4 \mathrm{Vac}| \mathrm{Co}$ tunnel junction, calculated by the ab initio KKR method. The moments of the Co halfcrystals are parallel.

show the occurrence of these hot spots in ab initio calculations for a Co $\mid$ Vacuum $\mid \mathrm{Co}$ junction using the KKR and TB-LMTO methods.

In the model calculation we assume a barrier with a constant potential of height $V_{\mathrm{B}}$ and thickness $D$. The potential $V(x, y, z)$ in the two halfcrystals is independent of $z$, the direction normal to the barrier. For the in-plane $x$ and $y$-dependence we assume a weak potential corrugation in the $x$-direction described by e.g., the Fourier coefficients $V_{G_{x}}$ and $V_{-G_{x}}$ for the smallest reciprocal lattice vectors in the $x$-direction. The eigenfunctions are then calculated in the nearly-free-electron approximation. In the following we discuss only the case of the perpendicular incidence $\mathbf{k}_{\|}=0$. In the unperturbed case, i.e., $V_{G_{x}}=0$, we obtain an incident wave $\mathrm{e}^{\mathrm{i} k_{z} z}$ with energy $E=k_{z}^{2}$ and a second wave $\mathrm{e}^{\mathrm{i} k_{z} z} \cos \left(G_{x} x\right)$ with energy $E=k_{z}^{2}+G_{x}^{2}$. For $V_{G_{x}} \neq 0$, but small, the new eigenfunctions, i.e., the Bloch waves, have still the same dominating plane wave character, either $e^{i k_{z} z}$ or $e^{\mathrm{i} k_{z} z} \cos \left(G_{x} x\right)$, but in addition a small admixture of the second plane wave component, so that the energy dispersion is only slightly changed. In addition we introduce two attractive $\delta$-functions $-\beta_{1} \delta(z+D / 2)$ and $-\beta_{2} \delta(z-D / 2)$ at the interfaces on both sides of the barrier, which allow us to introduce the interfaces states. The solution in the two halfcrystals and in the barrier are matched at the interfaces $z=-D / 2$ and $z=+D / 2$. If the $\beta_{1}$ (or $\beta_{2}$ ) value is sufficiently large, it introduces at the left (or right) interface an interface state for an energy below the bottom of each band. The interface state below the lower band $\left(k_{z}^{2}\right)$ is localized, while the one below the higher band $\left(k_{z}^{2}+G_{x}^{2}\right)$ is resonant, since it hybridizes weakly with the Bloch waves of the lower band $\left(k_{z}^{2}\right)$.
We now consider a Bloch wave $\mathbf{k}=\left(0,0, k_{z}\right)$ incident on the barrier. If the barrier is sufficiently high, the transition probability $|t|^{2}$ is relatively small, as is shown in Fig. 5a for energies away from the energy of the resonant interface states. In the vicinity of the resonance, we observe anomalies. The dashed curve gives the results for a single resonance on one side of the interface, obtained by allowing only $\beta_{1}$ or $\beta_{2}$ to be non-zero. At the resonance energy, we then obtain a maximum in the transmission, thus an enhanced tunneling probability which is due to the enhancement of the incoming wavefunction by the large amplitude of the resonant interface state. The following zero of $|t|^{2}$, an antiresonance, can be explained by the Fano effect [13]. While this behavior is as expected, a dramatic effect occurs in the case of two degenerate interface states on both sides of the barrier, created by two equal $\delta$-potentials $\beta_{1}=\beta_{2}$ We observe two resonance peaks, both showing full transmission, $|t|^{2}=1$, of the incident wave. The peaks are symmetrically situated at lower and higher energies compared with the energy of the single resonance, and both are accompanied by an antiresonance. The calculation of the wavefunctions shows, that for the lower peak both interface states form a bonding hybrid and for the higher peak an antibonding hybrid. Thus, for these resonances the total wavefunction is nearly symmetrical or antisymmetrical with respect to the center of the barrier, which directly explains the full transmission without any attenuation. Thus if the bonding-antibonding splitting $\Delta$ is larger than the natural resonance width $\Gamma, \Delta \gg \Gamma$, full transmission will
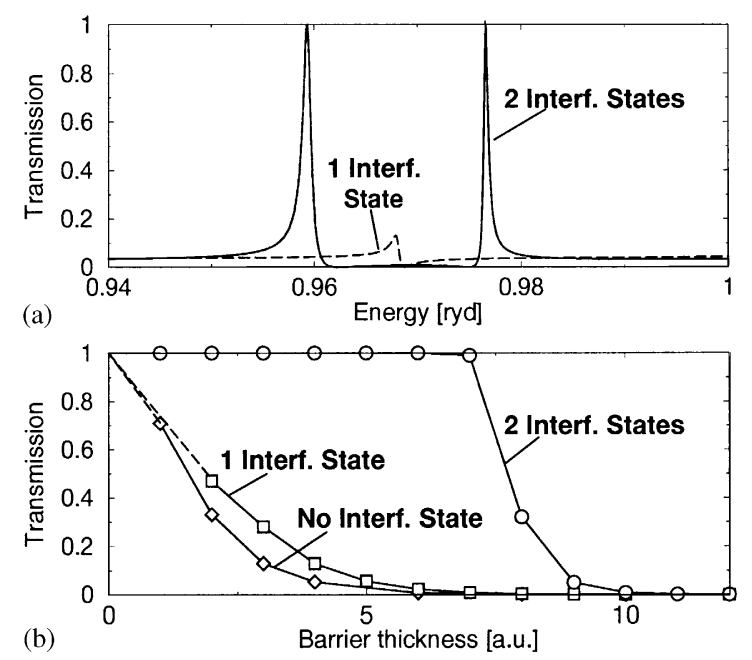

Fig. 5. Results of the analytical model. In Fig. 5a the transmission $|t|^{2}$ for a barrier with one interface state (dashed line) and for a symmetrical barrier with two interface states (solid line) is shown. In Fig. 5b the thickness dependence of the maximum transmission $|t|^{2}$ at the resonances for zero, one, and two interface states is shown. 
occur, while when both resonances fuse together, $\Delta \ll \Gamma$, the normal resonance behavior is observed. This can be understood in a time-dependent picture. If the lifetime $t_{\mathrm{R}}=\hbar / \Gamma$ of the resonance is much larger than the hopping time $t_{\mathrm{H}}=\hbar \pi / \Delta$ which the particle needs to coherently hop between the interface states on both sides of the barrier, then during the lifetime of the resonance, bonding and antibonding states can be formed, allowing the particle to fully penetrate the barrier via these hybrids. However, if $t_{\mathrm{R}} \ll t_{\mathrm{H}}$, this channel is no longer open and the incident wave is attenuated.

This effect shows up equally dramatically in Fig. 5b, where the maximum transmission probability $|t|^{2}$ at the resonance peak is plotted versus the barrier thickness $D$. In the case of the no interface state $\left(\beta_{1}=\beta_{2}=0\right)$, normal exponential dependence on the barrier thickness is obtained. For a single interface state on one side of the barrier, tunneling is somewhat enhanced, but basically the same attenuation is observed. On the other hand, for the symmetrical barrier with degenerate interface states, full transmission is obtained for small and intermediate thickneses and the exponential decay is strongly delayed. For very large thickneses one finds in all three cases the same exponential decrease, i.e., with the same decay length.

In the following, we will present a case study of resonance effects for the Co|Vacuum $\mid \mathrm{Co}\left(\begin{array}{lll}0 & 0 & 1\end{array}\right)$ barrier mentioned earlier. Contrary to the previous case, we vary the $\mathbf{k}_{\|}$-vector in the surface Brillouin zone (SBZ) and fix the energy at the Fermi level $E_{\mathrm{F}}$. We have calculated the transmittance $T\left(\mathbf{k}_{\|}, E_{\mathrm{F}}\right)$ by the ab initio TB-LMTO method, for which we refer to [14]. To resolve the spiky structures of the hot spots, a very large number (980 700) of $\mathbf{k}_{\|}$points in the irreducible part of the SBZ has been used. For the following results two FCC Co $\left(\begin{array}{lll}0 & 0 & 1\end{array}\right)$ halfcrystals have been considered, being separated by three layers of vacuum. For all $\mathbf{k}_{\|}$in the SBZ all eigenstates, if delocalized or localized, have been evaluated and analyzed by the transfer matrix method (for a recent application to multilayers see Ref. [15]). Here we consider only the parallel alignment of the Co moments and the conductance in the minority band.

Let us discuss in more detail the structure of the four dominating double-spots in Fig. 4, which occur along the $\bar{\Gamma}-\bar{X}$ line, i.e., the diagonal $k_{x}=k_{y}$. The isointensity contour of the transmittance is shown in Fig. 6, where darker areas denote higher intensities. For symmetry reasons the contours show mirror symmetry with respect to the $\bar{\Gamma}-\bar{X}$ line. All the isointensity lines merge together in two points on this line $k_{x}^{ \pm}=k_{y}^{ \pm}=$ $(2.485172 \pm 0.000167) / a$, where $a$ is the lattice constant of the FCC Co. As indicated by the vanishing linewidth at these points, these are localized states which do not contribute to the current. At these two points they coexist with two incident states with full transmission. The wavefunctions of the two localized states are bonding and antibonding combinations of the surface

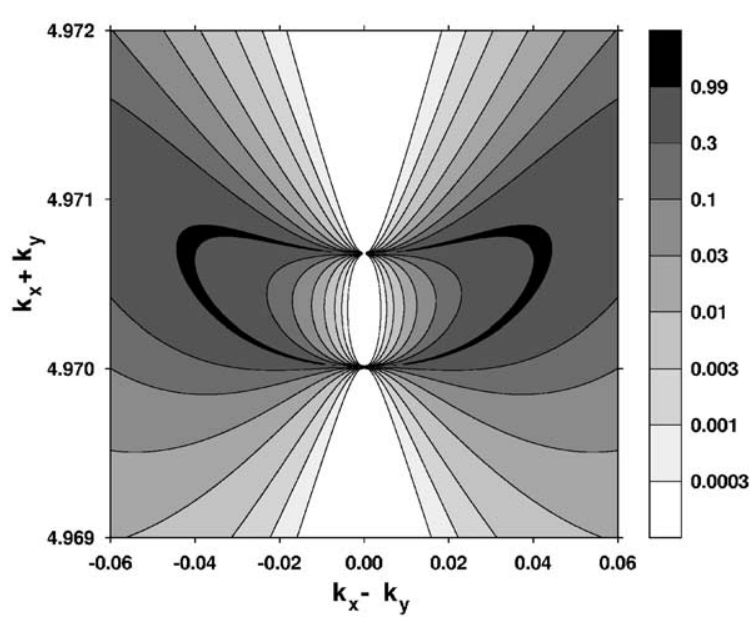

Fig. 6. Enlarged transmission plot for the minority band of $\mathrm{Co} / 3 \mathrm{Vac} \mid \mathrm{Co}$ tunnel junction, calculated by the ab initio TBLMTO method. The moments of the Co halfcrystals are parallel aligned. Darker areas correspond to higher transmission probabilities. The reciprocal lattice vectors are given in units of $1 / a$, where $a$ is the lattice constant of the FCC Co.

states of the two Co halfcrystals. In fact the localization is symmetry enforced: the localized state is symmetric with respect to a reflection at the $\bar{\Gamma}-\bar{X}$-axis while all propagating states for this $\mathbf{k}_{\|}$-point are antisymmetric. However, for a small deviation from the diagonal, other states are intermixed, leading to the loss of orthogonality and to a transition from a localized to a resonant state with a small, but finite halfwidth. The darkest contour area, indicating a transmittance larger than 0.99 , contains a line with full transmission 1. With increasing deviation from the diagonal the linewidth increases and the two resonance join together into a single resonances, so that for larger distances the transmittance decreases.

As we have demonstrated above, large effects from interface resonances in general require a symmetrical barrier, since a one-sided resonance is less effective for the tunneling process. It is the formation of bonding and antibonding states, which makes the barrier transparent. Therefore, we believe that interface roughness as well as a finite bias voltage will reduce the importance of hot spots. However, since this is a quantitative question, reliable calculations would be highly desirable.

In summarizing, we evaluated by model and ab initio calculations the effects of interface states on the conductance of magnetic tunnel junctions. The hot spots found by different groups in ab initio calculations can be explained by the formation of bonding and antibonding hybrids between the interface states on both sides of the barrier. If the resonance condition for these hybrid resonances is met, the electron can tunnel 
through the barrier without attenuation. Surface states on one side of the interface influence the conductance to a smaller degree. Since full transmission is only obtained for a symmetrical barrier, we expect interface roughness and finite bias voltages to reduce the importance of hot spots and interface states in general.

Financial support for this work was provided by the RT Network Computational Magnetoelectronics (Contract RTN1-1999-00145) and the TMR-Network Interface Magnetism (Contract ERBFMRXCT 960089) of the European Commission, the Grant Agency of the AS CR (A1010829), the Grant Agency of the Czech Republic (202/00/0122), the Ministry of Education of the Czech Republic (COST P5.30) and the Scientific and Technological Cooperation between Germany and the Czech Republic (TSR-013-98).

\section{References}

[1] T. Miyazaki, N. Tezuka, J. Magn. Magn. Mater. 139 (1995) L231.
[2] J.S. Moodera, L.R. Kinder, T.M. Wong, R. Meservey, Phys. Rev. Lett. 74 (1995) 3273.

[3] W.H. Butler, X.-G. Zhang, X. Wang, J. van Ek, J.M. MacLaren, J. Appl. Phys. 81 (1997) 5518.

[4] I.I. Oleinik, E.Yu. Tsymbal, D.G. Pettifor, Phys. Rev. B 62 (2000) 3952.

[5] J.M. MacLaren, X.-G. Zhang, W.H. Butler, Xindong Wang, Phys. Rev. B 59 (1999) 5470.

[6] K. Wang, P.M. Levy, S. Zhang, L. Szunyogh, P. Weinberger. Phys. Rev. B, submitted for publication.

[7] Ph. Mavropoulos, N. Papanikolaou, P.H. Dederichs, Phys. Rev. Lett. 85 (2000) 1088.

[8] V. Heine, Proc. Phys. Soc. 81 (1962) 300.

[9] V. Heine, Surf. Sci. 2 (1964) 1.

[10] W.H. Butler, X.-G. Zhang, T.C. Schulthess, J.M. MacLaren, Phys. Rev. B. 63 (2001) 54416.

[11] J. Mathon, A. Umerski, Phys. Rev. B 63 (2001) R220403.

[12] P. Zahn, presented at RT Workshop "GMR and TMR", Dresden, December 2000.

[13] U. Fano, Phys. Rev. 124 (1961) 1866.

[14] J. Kudrnovský, V. Drchal, C. Blaas, P. Weinberger, I. Turek, P. Bruno, Phys. Rev. B 62 (2000) 15084.

[15] T. Kostyrko, Phys. Rev. B. 62 (2000) 2458. 